\title{
Understanding Zika virus as an STI: findings from a qualitative study of pregnant women in the Bronx
}

\author{
Miguel Rodriguez (), ${ }^{1}$ Ayla Lord, ${ }^{2}$ Carolina C Sanabia, ${ }^{2}$ Abigail Silverio, ${ }^{2}$ \\ Meleen Chuang, ${ }^{2}$ Siobhan M Dolan ${ }^{1,2}$
}

\begin{abstract}
${ }^{1}$ Albert Einstein College of Medicine, Bronx, New York, USA ${ }^{2}$ Obstetrics \& Gynecology and Women's Health, Montefiore Health System, Bronx, New York, USA
\end{abstract}

Correspondence to Dr Siobhan M Dolan, Albert Einstein College of Medicine, Bronx, NY 10461, USA; sdolan@montefiore.org

Received 26 April 2019 Revised 2 July 2019 Accepted 8 July 2019 Published Online First 26 July 2019

\section{TRACT}

Objectives Pregnant women in the Bronx were at risk for travel-related Zika exposure in the USA between 2016 and 2017. This qualitative study explored the experiences of 13 pregnant women to learn about their knowledge of Zika and prevention measures.

Methods In the summer of 2017, pregnant women at risk of travel-related Zika exposure were interviewed in either Spanish or English to learn about their experiences and transcripts were analysed using a grounded theory approach.

Results Most participants were Latinas living in the Bronx, median age of 29 years and median household income between $\$ 26000$ and $\$ 50000$. Participants displayed a strong understanding of Zika transmission via mosquito bites yet lacked knowledge about its sexual transmission. Interviews revealed three key themes: (1) Zika as a new disease, (2) denial as a coping mechanism and (3) the recommendation to treat Zika as an STI. Women observed Zika as a brand new disease with early messages emphasising mosquito-borne transmission. They lacked awareness of newer messaging about sexual transmission. Furthermore, if women did read about risk of sexual transmission, many stated being in denial and struggling with recommendations to prevent sexual transmission. Barriers included problems changing travel plans and rejection of condom use. Women unanimously suggested labelling Zika as an STI and adding it to existing lists of STIs for messaging and outreach in community-based and clinical prevention.

Conclusion Many pregnant women were unaware that Zika virus can be sexually transmitted due to: (1) novelty of Zika, (2) denial as a coping mechanism and (3) Zika not being listed along with well-known STIs. Overcoming these barriers via community-based as well as clinical education for pregnant women in the Bronx would be helpful in 2019 and beyond when the risk of travelrelated Zika exposure remains a public health threat to optimal pregnancy outcomes.

\section{BACKGOUND}

Zika virus infection during pregnancy presents a serious teratogenic risk for pregnant women. ${ }^{1}$ Infection causes a mild illness with $20 \%$ of affected individuals showing rash, conjunctivitis, fever and/or arthralgia. Fetal effects of congenital Zika include severe microcephaly with partially collapsed skull, thin cerebral cortices with subcortical calcifications, macular scarring and focal pigmentary retinal mottling, congenital contractures and marked early hypertonia with symptoms of extrapyramidal involvement. ${ }^{2}$ The outbreak in the Americas began in early 2015 when Zika was first reported in Brazil and linked with rapid increase in microcephaly reported among infants born in Northeastern Brazil. ${ }^{3}$ From Brazil, Zika spread throughout Latin America and the Caribbean with a current world map indicating endemic areas available through Centers for Disease Control and Prevention (CDC) at https://wwwnc. cdc.gov/travel/page/zika-information.

In 2017, the United States had a total of 452 reported cases of symptomatic Zika including vector based and sexual transmission. ${ }^{4}$ New York State had the second highest number of cases among the 50 states, following Florida. ${ }^{4}$ Travel-associated Zika infection was reported to the New York City Department of Health in 130 cases in $2017 .{ }^{5}$ Most at risk were pregnant women who travelled or whose partners travelled to affected areas in the Caribbean or South or Central America. The Bronx had the highest number of reported cases of any borough with 46 cases. $^{5}$

The Bronx is a diverse community with $51.7 \%$ of the population reporting Hispanic ancestry, mostly from Puerto Rico (36.4\%) and the Dominican Republic (39.4\%) . ${ }^{6}$ Both areas had active Zika transmission and CDC recommends that pregnant women avoid travel or employ enhanced precautions if travelling there. ${ }^{78}$ About 21249 births occur every year in the Bronx, with the majority (12 360) among Hispanics. ${ }^{9}$ Many other immigrant groups from countries with high level of Zika risk, such as Guyana, Jamaica and Mexico, also live in the Bronx. ${ }^{6}$

\section{OBJECTIVE}

In the summer of 2017 , we undertook a qualitative study of pregnant women in prenatal care at Montefiore Medical Center in the Bronx, New York, seeking to understand the experiences of pregnant women at risk of Zika exposure. The goal was to learn about women's knowledge of Zika and understand their experiences with Zika education and prevention. Of interest, we asked about the sexual transmission of Zika and present the findings on this topic.

\section{METHODS}

\section{Recruitment}

Participants were recruited from the Eastchester Professional Center at Montefiore Medical Center when they were seen for counselling and testing for prenatal Zika infection. Women who reported possible Zika exposure due to travel to areas with 
high risk by themselves or their partners were asked to participate. After their provider ordered Zika diagnostic testing following CDC guidelines, they were approached and asked to participate. CDC guidelines changed halfway throughout the study on 24 July 2017, to recommend testing only for pregnant women with potential Zika exposure that were symptomatic. ${ }^{10}$ However, local New York City testing guidelines did not change at that time (they later changed in October 2017), and thus, recruitment for the study stayed consistent through the summer of 2017. Women eligible to participate in the study were pregnant, 18 years or older and able to speak English or Spanish well enough to participate in an interview lasting approximately $20 \mathrm{~min}$. All participants provided written informed consent, completed a questionnaire providing demographic information and then participated in a face-to-face interview that was recorded. Participants were given a $\$ 40$ gift card as compensation for their time. The study was approved by the Institutional Review Board at Albert Einstein College of Medicine and Montefiore Medical Center, and precautions to protect human subjects were followed throughout the study. All women were informed that participation in the study was optional.

\section{Interviewing}

The Zika clinical research team included the PI who is an $\mathrm{Ob} /$ Gyn clinical geneticist (SMD), a medical student (MR), an MPH student (AL), a college student (AS) and a research assistant (CCS). The team included three fluent Spanish speakers (CCS, AS and MR). Four members of the team (MR, AL, AS and CCS) conducted the semistructured interviews $(n=13)$ in person. Nine interviews were conducted in English and four in Spanish based on the participant's language preference. The interview guide included recommended questions to gather information on the participants' knowledge, attitudes and experiences. Participants were asked about their general knowledge of Zika infection, as well as their attitudes towards and experiences with Zika education, prevention, counselling and testing. In addition, women were asked to give their recommendations on how Zika prevention could be improved in their community.

\section{Data analysis}

All interviews were audio-recorded and transcribed verbatim. Interviews conducted in Spanish were translated into English then transcribed. Weekly team meetings reviewed interviews performed and refined the interview script. Preliminary analysis of emerging topics included listening to audio recordings, extracting key quotes, listing emerging themes and noting new concepts. A preliminary codebook was begun that captured themes for later analysis of transcripts with Dedoose, 'a web application for managing, analysing, and presenting qualitative and mixed method research data'. ${ }^{11}$ Transcripts were then analysed by the research team using a grounded-theory method. ${ }^{12}$ In the analysis, repeating ideas, words and phrases used regularly were identified as codes. Subsequently, these codes were organised into themes, which further identified overriding concepts. Each transcript was coded individually in Dedoose, by at least four members of the team, to develop a list of codes and themes. After 13 interviews were conducted, saturation was noted as no new themes were identified.

\section{RESULTS}

From 13 July to 14 August 2017, 13 participants provided informed consent to participate in the study, completed the questionnaire and were interviewed. Interviews took a median time of $31 \mathrm{~min}$. Participants had a median age of 29 years, median number of pregnancies was 3 and the median number of children was 1 . Table 1 provides the demographic characteristics of the participants.

\section{THEMES}

Participants' comments revealed excellent knowledge about Zika transmission via a mosquito bite.

- 'I know it's a virus and transmitted by a mosquito bite' (25, travelled to Ecuador).

- 'Yes, I saw on television that they were saying that Zika is a disease transmitted by a mosquito, and how when a pregnant woman gets it, the baby may have problems' $(29$, travelled to Dominican Republic).

However, there was a general lack of knowledge among participants about the sexual transmission of Zika. Analysis identified three main themes: (1) Zika as a new disease, (2) denial as a coping mechanism and (3) the need to treat Zika as an STI.

\section{Lack of knowledge regarding sexual transmission of Zika}

A general lack of knowledge about sexual transmission of Zika in the community was revealed. Many participants did not know Zika could be sexually transmitted and many women reported that their friends and family were unaware as well.

- '... when I talk to my friends, a lot of them they don't know that it can be sexually transmitted' (25, travelled to Ecuador).

- 'I didn't even know it would - you could transmit sexually. That I didn't know. I thought it was like only female' (30, travelled to Puerto Rico).

- 'They [friends] knew way more than I did [about Zika] but they didn't know that it can be sexually transmitted' (28, husband travelled to Puerto Rico).

Participants expressed concern about this lack of knowledge and expressed a desire to know more about Zika's sexual transmission.

- 'People don't know Zika's an STD and that's concerning ... that should be common knowledge that people know. It is an STD. I don't think that's out there like that and that's really sad' (30, travelled to Saint Thomas).

- 'They should make it very known, just the same way they make HIV known and sexually transmitted and anything else that comes out they should do the same' (28, husband travelled to Puerto Rico).

- 'It's not talked about. They will test you for AIDS quicker than Zika. And Zika is an epidemic outside the country. And that's concerning' (28, husband travelled to Puerto Rico).

For one participant it was the first time she had ever seen information about sexual transmission of Zika. Other women echoed this sentiment, stating how they had never seen information printed about how to prevent sexual transmission of Zika.

Even if the participants knew about sexual transmission, many did not know either how to protect themselves or how long to use preventive measures. Many participants found out at the last minute, right before a trip, creating inconveniences such as travelling for a honeymoon. In fact, many situations arose where the participants or their partners had to travel, either due to family obligations, work or because of trips planned in advance.

- '... before I actually went, I was warned and told not to go, but ... I couldn't do that because I went for a funeral' (21, travelled to Honduras).

- 'Cause you can't not travel. There are certain places such as weddings, funerals, visiting relatives, things like that, that 


\section{Table 1 Demographic characteristics of participants}

\begin{tabular}{|c|c|c|c|c|c|}
\hline Variable & $\mathrm{N}$ & $\%$ & Variable & Median & $\begin{array}{l}\text { Range } \\
\text { (min, } \\
\max )\end{array}$ \\
\hline Gender & & & \# of pregnancies & 3 & $(1,6)$ \\
\hline Female & 13 & 100 & \# of kids & 1 & $(0,2)$ \\
\hline Ethnicity & & & Age (years) & 29 & $(18,39)$ \\
\hline Latino/Hispanic & 12 & 92 & & & \\
\hline $\begin{array}{l}\text { Non-Latino/non- } \\
\text { Hispanic }\end{array}$ & 1 & 8 & & & \\
\hline \multicolumn{6}{|l|}{ Self-reported race } \\
\hline $\begin{array}{l}\text { Black/African- } \\
\text { American and white }\end{array}$ & 4 & 30 & & & \\
\hline White & 1 & 8 & & & \\
\hline $\begin{array}{l}\text { American Indian/ } \\
\text { Alaskan native }\end{array}$ & 1 & 8 & & & \\
\hline $\begin{array}{l}\text { Black/African- } \\
\text { American and } \\
\text { American Indian/ } \\
\text { Alaskan native }\end{array}$ & 1 & 8 & & & \\
\hline $\begin{array}{l}\text { Native Hawaiian/ } \\
\text { Pacific Islander }\end{array}$ & 1 & 8 & & & \\
\hline Other/did not respond & 5 & 38 & & & \\
\hline \multicolumn{6}{|l|}{ Language } \\
\hline Spanish & 4 & 30 & & & \\
\hline English & 9 & 70 & & & \\
\hline \multicolumn{6}{|l|}{ Civil status } \\
\hline Married & 7 & 54 & & & \\
\hline Cohabiting & 4 & 30 & & & \\
\hline Single & 2 & 16 & & & \\
\hline \multicolumn{6}{|l|}{ Education } \\
\hline GED & 1 & 8 & & & \\
\hline Some college & 7 & 54 & & & \\
\hline College degree & 4 & 30 & & & \\
\hline Graduate degree & 1 & 8 & & & \\
\hline \multicolumn{6}{|l|}{$\begin{array}{l}\text { Estimated annual } \\
\text { household income }\end{array}$} \\
\hline Less than $\$ 25000$ & 4 & 30 & & & \\
\hline$\$ 26000-\$ 50000$ & 1 & 8 & & & \\
\hline$\$ 51000-\$ 80000$ & 2 & 16 & & & \\
\hline Greater than $\$ 80000$ & 3 & 23 & & & \\
\hline Refused/other & 3 & 23 & & & \\
\hline \multicolumn{6}{|l|}{ Employment status } \\
\hline Full time & 6 & 47 & & & \\
\hline Part-time & 4 & 30 & & & \\
\hline Unemployed & 3 & 23 & & & \\
\hline \multicolumn{6}{|l|}{ Type of insurance } \\
\hline Medicaid & 8 & 62 & & & \\
\hline Medicare & 1 & 8 & & & \\
\hline Commercial & 4 & 30 & & & \\
\hline \multicolumn{6}{|l|}{ County of residence } \\
\hline Bronx & 11 & 84 & & & \\
\hline Manhattan & 1 & 8 & & & \\
\hline Rockland & 1 & 8 & & & \\
\hline \multicolumn{6}{|l|}{ Zika tested } \\
\hline Yes & 13 & 100 & & & \\
\hline \multicolumn{6}{|l|}{ Zika test results } \\
\hline Negative & 13 & 100 & & & \\
\hline
\end{tabular}

you can't stop because of certain outbreaks in demographics, you just have to be careful' (30, travelled to Saint Thomas).

\section{New disease and evolution of Zika}

Women reported that Zika being a 'new' disease affected their lack of knowledge of its sexual transmission.

- 'In my head, Zika's brand new. It's a brand new disease that was just introduced to a lot of Americans. I didn't categorize it yet. I didn't put it together' $(28$, husband travelled to Puerto Rico).

Due to the novelty, people did not know how to label or classify Zika in their head. Zika was different from other diseasesasymptomatic in the pregnant mother but teratogenic for the developing fetus. This caused cognitive dissonance for some participants and a barrier to understanding the full range of modes of transmission.

- 'I wouldn't think of a disease that came from another country to be a sexually transmitted disease' (28, husband travelled to Puerto Rico).

- 'That I didn't know. I thought it was like only female' (30, travelled to Puerto Rico).

- 'But now, knowing, it's like it affects not just pregnant women, it affects anyone, and its also sexually transmitted... seeing that as a virus' (30, travelled to Saint Thomas).

- 'But just STD affects you. It (Zika) affects the baby. So, that's-that's scary' (25, travelled to Ecuador).

Initial messages focused on mosquito-borne transmission and avoidance of travel and women reported being very familiar with these messages. Over time, it was revealed that Zika was sexually transmitted, and this was challenging because it required women to continue to process and add new information.

- 'All you always hear is that a mosquito bites you and you get the virus' (25, travelled to Ecuador).

- 'Yeah. Actually, fliers are saying that those are transmitted from mosquitoes but now they're saying it could be transmitted sexually as well' (30, travelled to Puerto Rico).

This novelty and confusion around Zika transmission became especially apparent when participants compared it to HIV.

- 'It's already common knowledge that AIDS is a sexually transmitted disease but is that the only way you can contract it? No. You can get it through needles, you can get it through other means, but that's the most popular way. Just like with Zika. The most popular way that people know of having Zika spread is through mosquitoes. That is not the only way, you know? So ... that should be common knowledge that people know' (30, travelled to Saint Thomas).

\section{Denial as a coping mechanism}

Several participants were hesitant to acknowledge information about Zika's sexual transmission stating they or their friends would either ignore or deny this information.

- 'Cause people are like, "Oh ...", you know, “...mosquitoes. I don't go outside anyways". You know, they'll kind of ignore the fact that it's more than that' $(30$, travelled to Saint Thomas).

- 'Cause honestly, I did read - I didn't read the sexually transmitted part until they told me ... actually, I did read it but I think it was like a glance over because I didn't know, like the symptoms. So I didn't like, fully understand' (30, travelled to Saint Thomas).

- 'Maybe I shied away from it so much, that it was sexually transmitted disease or something like that' (28, husband travelled to Puerto Rico). 
One reason for coping through denial was that it allowed women to avoid the recommendation to use condoms or abstain from sexual intercourse. Participants presented a variety of responses to the recommendations to use condoms.

- 'I mean in my religion, it goes against the religion but for safety reasons, I feel that it should be ok' $(25$, travelled to Ecuador).

- 'No. It wouldn't be a problem because I wouldn't use them [Condoms]' (29, travelled to Dominican Repubic).

- 'I mean I'm married and that will be a problem in my marriage. If I tell my husband that we're never gonna have no sex because I'm worried about that [Zika transmission]. He will be arguing that I'm denying myself' (25, travelled to Ecuador).

Responses revealed the importance of factors such as partner acceptance and overcoming fear as crucial for prevention of sexual transmission of Zika. Risk of sexual transmission of Zika advances an understanding that Zika infection is not just a female thing' but rather now incorporates a social dynamic with sexual partners, which women may hesitate to embrace.

\section{Treat Zika like an STI}

Women were asked to give recommendations for community education about sexual transmission of Zika. The overwhelming response was to treat it like an STD.

- 'Treat it like if it was an STD' (25, travelled to Ecuador).

- 'I think we're gonna reach it more ... just as add it on as [an] STD... I think the fact that we were planning [to be pregnant] helped but maybe it's a good thing to mention to those that are not planning or just like that are in relationships and just having sex. They should know this - this part of like an STD' (25, travelled to Ecuador).

- 'It's something really serious ... it should be as severe as the STD' (25, travelled to Ecuador).

- 'It's an STD, like that is huge' (30, travelled to Saint Thomas). Women expressed many ways for Zika to be labelled and advertised as an STI.

- 'On a poster? Like, the newest form of an STD is Zika' (30, travelled to Saint Thomas).

- "What do you mean Zika is an STD?" Yeah it's not a mosquito bite. Or like, I will put like... "Not Just a mosquito bite," you know?' (30, travelled to Saint Thomas).

They also recommended adding it to the list of STDs:

- 'Include it in one of the bulletins like chlamydia, herpes, the Zika virus' (39, partner travelled to Mexico).

- No I think it should be added to the same list of STDs... like when they say AIDS, Hepatitis, Syphilis, all those, Zika should be on there. It's not on there' (30, travelled to Saint Thomas).

\section{DISCUSSION}

This qualitative study revealed an emerging understanding of Zika transmission and prevention among pregnant women in the Bronx. In the summer of 2017, women suggested their understanding of Zika transmission and prevention was in a transition between the acute public awareness of the mosquito-borne epidemic that began in 2016 to the chronic nature of preventing Zika infection both through travel-related and sexual transmission that continues to this day. Analysis of the interviews we conducted identified three main themes as part of this transition: Zika as a new disease, denial as a means of coping and the need to treat Zika as an STI.
Table 2 Community advice for public health workers and healthcare practitioners for promoting prevention of sexual transmission of Zika

\begin{tabular}{l} 
Messaging for Prevention of Sexual Transmission \\
\hline Advice
\end{tabular}

Zika was a new infection in the Americas in 2016, and the pregnant women we interviewed were well aware of its transmission via mosquito bites, its potentially harmful impact on pregnancy and recommendations for travel restrictions to avoid exposure. Zika infection during pregnancy was particularly worrisome because of its stealth nature: it can infect an adult woman without symptoms but cause a teratogenic syndrome for her developing fetus.

Risk of sexual transmission of Zika was not well appreciated by the women we interviewed. Furthermore, transmission of this new infection both through mosquito bites and sexually created a barrier to understanding for women because it could not be placed neatly into a category that was previously well understood. Women found it hard to understand an infection that was first understood to be transmitted by mosquitos but then was also sexually transmitted.

Furthermore, when presented with information about the sexual transmission, most participants presented difficulties with the measures recommended to prevent sexual transmission of Zika infection. Avoidance and denial were commonly invoked when dealing with this information. Preventive measures such as abstinence or using condoms in the context of a monogamous relationship, to protect the fetus from infection, were not well received both by pregnant women and their partners. The timing of the information, which was largely conveyed at prenatal care visits in the medical setting, was also a barrier because women were often travelling to areas with a risk of Zika exposure for their honeymoons, vacations and to visit family. These were felt to be times that abstinence or condom use were particularly burdensome.

These findings suggest that community-based messaging about the sexual transmission of Zika infection and the ways to prevent transmission would be quite helpful to women and families. These messages should not be exclusively targeted to women but should also include partners and community leaders. This community-based health promotion could yield great results especially because most of the women interviewed wanted to help address this lack of knowledge in their communities and they wanted to attain this knowledge much earlier than the day before they were leaving on a trip. Confining education to the medical setting is also limiting and women 
told us that the use of social media, including pregnancy related apps, would allow broader community-based educational opportunities.

The Bronx has one of the highest burdens of STIs in the USA, ${ }^{13}$ and many of the women interviewed had a strong familiarity with community-based messaging about STI prevention from medical visits with healthcare providers, public health posters or fliers, or even from school. However, women pointed out that they did not understand why Zika was not included in these familiar outlets. It was not on a routine checklist for STIs and women told us that it should be. The 'Get Tested' campaign for HIV prevention is advertised on billboards and buses in the Bronx, and many of the women we interviewed told us that Zika prevention efforts should be advertised in the same way. Even though Zika was perceived to be a new disease, future public health messaging could gain further reach by making use of preestablished campaigns in the community and making comparisons with similar STIs that are already well understood (see table 2). As one participant told us regarding Zika prevention, 'Treat it like if was an STD'.

As the Zika epidemic of 2016 fades into the background, Zika infection during pregnancy continues to pose a threat to pregnant women and fetuses through travel-related exposure. Women in this study provided educational recommendations that included powerful phrases for new public health campaigns or education such as, 'Not just a female thing', 'Not just a mosquito thing' and 'The newest form of an STD'. This enthusiasm and energy present a roadmap for community-based outreach and education about the sexual transmission of Zika in the Bronx and perhaps in other communities with high numbers of women at risk.

\section{Key messages}

- Pregnant women in the Bronx reported being aware of Zika's transmission via mosquito bites and were aware of guidance to avoid travel to affected areas while pregnant.

- Pregnant women in the Bronx reported limited awareness of Zika virus being an STI and reported cognitive dissonance with that concept.

- Recommendations on how to promote prevention of sexual transmission of Zika both in clinic and through communitybased messaging to best reach women and their families.

Handling editor Anna Maria Geretti
Contributors Conception and design of the work (MR and SMD). Acquisition, analysis and interpretation of data (all authors). Drafting the work (MR and SMD). Revising critically for important intellectual content and final approval of the version published (all authors). Agreement to be accountable for all aspects of the work in ensuring that questions related to the accuracy or integrity of any part of the work are appropriately investigated and resolved (SMD).

Funding The authors have not declared a specific grant for this research from any funding agency in the public, commercial or not-for-profit sectors.

Competing interests None declared.

Patient consent for publication Not required.

Ethics approval IRB Number: 2017-7845

Provenance and peer review Not commissioned; externally peer reviewed.

Data availability statement All data relevant to the study are included in the article or uploaded as supplementary information.

ORCID iD

Miguel Rodriguez http://orcid.org/0000-0003-1661-4448

\section{REFERENCES}

1 Rasmussen SA, Jamieson DJ, Honein MA, et al. Zika virus and birth defects reviewing the evidence for causality. N Engl J Med Overseas Ed 2016;374:1981-7.

2 Moore CA, Staples JE, Dobyns WB, et al. Characterizing the pattern of anomalies in congenital Zika syndrome for pediatric clinicians. JAMA Pediatr 2017;171:288-95.

3 Dyer 0 . Zika virus spreads across Americas as concerns Mount over birth defects. BMJ 2015;351.

4 Centers for Disease Control and Prevention. 2017 case counts in the US. Available: https://www.cdc.gov/zika/reporting/2017-case-counts.html [Accessed 26 Apr 2019].

5 NCY. New York City department of health and mental hygiene. Available: http:// www1.nyc.gov/site/doh/health/health-topics/zika-virus.page [Accessed 26 Apr 2019].

6 Statistical Atlas. Bronx County, sub headings: race and ethnicity, ancestry, National origin. Available: https://statisticalatlas.com/county/New-York/Bronx-County/Ancestry [Accessed 26 Apr 2019].

7 CDC. Cdc travel alerts for Zika - Dominican Republic. Available: https://wwwnc.cdc. gov/travel/notices/alert/zika-virus-dominican-republic [Accessed 26 Apr 2019].

8 CDC. Cdc travel alerts for Zika - Puerto Rico. Available: https://wwwnc.cdc.gov/travel/ page/risk-of-zika-selected-destination [Accessed 26 Apr 2019].

9 NY gov. Bronx County health indicators by Race/Ethnicity, 2012-2014. Available: https://www.health.ny.gov/statistics/community/minority/county/bronx.htm [Accessed 26 Apr 2019].

10 Oduyebo T, Polen KD, Walke HT, et al. Update: interim guidance for health care providers caring for pregnant women with possible Zika virus exposure - United States (including us territories), July. MMWR Morb Mortal Wkly Rep 2017;2017:781-93.

11 SocioCultural Research Consultants, LLC. Dedoose version 7.0.23, web application for managing, analyzing, and presenting qualitative and mixed method research data, 2016. Los Angeles, Ca. Available: www.dedoose.com

12 Auerbach C, Silverstein L. Qualitative data: an introduction to coding and analysis. New York: New York University Press, 2003.

13 NY gov. Sexually transmitted infections surveillance report New York state, 2017. Available: https://www.health.ny.gov/statistics/diseases/communicable/std/docs/sti_ surveillance_report_2017.pdf [Accessed 26 Apr 2019]. 